\title{
On the Equivalence of Least Costly and Traditional Experiment Design for Control
}

\author{
Cristian R. Rojas a Juan C. Agüero a James S. Welsh ${ }^{\text {a }}$ Graham C. Goodwin ${ }^{\mathrm{a}}$ \\ ${ }^{a}$ School of Electrical Engineering and Computer Science, The University of Newcastle, NSW 2308, Australia.
}

\begin{abstract}
In this paper we establish the equivalence between least costly and traditional experiment design for control. We consider experiment design problems for both open and closed loop systems. In open loop, equivalence is established for three specific cases, relating to different parametrisations of the covariance expression (i.e. finite and high order approximations) and model structure (i.e. dependent and independently parameterised plant and noise models). In the closed loop setting, we consider only finite order covariance expressions. $\mathcal{H}_{\infty}$ performance specifications for control are used to determine the bounds on the covariance expression for both the open and closed loop cases.
\end{abstract}

Key words: Optimal input design, identification for control, experiment design.

\section{Introduction}

An important issue in system identification is that of experiment design, i.e. how should one perturb the system to obtain maximal information (Fedorov, 1972; Whittle, 1973; Goodwin and Payne, 1977). Considerable work has been published in the engineering literature on this topic, focussed mainly on experiment design for dynamic systems (Levadi, 1966; Mehra, 1974; Goodwin and Payne, 1977; Zarrop, 1979; Gevers, 2005; Rojas et al., 2007). A primary motivating factor for system identification and hence experiment design, is to obtain accurate plant models for use in one of the many model based control system design methodologies. To this end there has been a substantial amount of recent work aimed specifically at investigating experiment design for control (Jansson, 2004; Hildebrand and Gevers, 2003; Hjalmarsson, 2005; Gevers and Bombois, 2006).

In the more recent research on experiment design for control, a new paradigm has been proposed, namely Least Costly Identification Experiments for Control (Bombois

\footnotetext{
ऋ This paper was not presented at any IFAC meeting. Corresponding author C. R. Rojas. Tel. +61-2-492 16151. Fax +61-2-496 01712.

Email addresses:

Cristian.Rojas@studentmail.newcastle.edu.au (Cristian R. Rojas), Juan. Aguero@newcastle.edu.au (Juan C. Agüero), James.Welsh@newcastle.edu.au (James S. Welsh), Graham. Goodwin@newcastle.edu.au (Graham C. Goodwin).
}

et al., 2004a,b, 2006). The objective of this paradigm is to design an experiment based on a robust control performance specification with respect to quality constraints on the estimated model and which incurs the smallest possible cost (Bombois et al., 2006). The cost considered is generally associated with the input and/or output power, or the duration of the experiment. This least costly experiment design formulation can be contrasted with the traditional approach where one determines the optimal experiment that minimises, for example, a control oriented measure of the model accuracy, subject to input and/or output power constraints.

Least costly open loop experiment design for control (Bombois et al., 2004a) seeks to minimise the input power under the constraint that the controller, designed based on the identified model, is guaranteed to stabilise and achieve a desired $\mathcal{H}_{\infty}$ performance on the true system. This is accomplished in a two step procedure. The first step determines the size of the identified model uncertainty that can be tolerated which, when used for the design of a controller, will satisfy the $\mathcal{H}_{\infty}$ performance specifications. The least powerful input signal is then designed in the second step such that the identified model uncertainty is less than that determined in the first step.

In the closed loop case (Bombois et al., 2006), the cheapest experiment is considered to be that which minimises the impact of the perturbation as observed on either the input or output of the system. A methodology, which is analogous to that used in the least costly open loop case, is then used for experiment design. 
The results of Bombois et al., 2004a, 2006, 2004b are based on variance errors only (although the least costly paradigm has been recently extended to also include bias errors (Bombois and Gilson, 2006)). The early results (Bombois et al., 2004b) use variance expressions which are asymptotic in the model order and data length. Recent results (Bombois et al., 2004a, 2006, 2004b) are based on more accurate parameter covariance expressions which are non-asymptotic in the model order (Ljung, 1999, Section 9.4).

The identification procedure considered in the least costly approach to experiment design is the Prediction Error Method (PEM). In the closed loop case the results have been specifically developed for the direct identification method.

In this paper, we explore the relationship between two approaches to experiment design, which differ in the way the input/output power is considered as part of the optimisation.

We will say that a given experiment design formulation is in the traditional framework if the input/output power is included as a constraint in the optimisation problem, so that the purpose of the optimal experiment is to maximise a given quantity related to the model quality, i.e. a function of the parameter covariance matrix, under an input/output power constraint.

On the other hand, a least costly experiment design formulation is defined as an optimisation problem where the input/output power is minimised subject to a model quality constraint, given as a function of the parameter covariance matrix.

In (Bombois et al., 2006) the least costly paradigm is stated to be a 'dual approach' to the traditional optimal experiment design problem. The aim of the current paper is to establish this equivalence between the two paradigms and hence show that they are indeed dual problems. Specifically, we show equivalence between the traditional optimal experiment design problem and the results for the least costly approach for both open and closed loop systems. Since there is no unification between the open and closed loop cases with respect to a measure of the model quality, we establish equivalence for each of the cases previously analysed in the least costly framework (Bombois et al., 2004a,b, 2006).

The results presented in the paper establish that solutions of several experiment design problems in the least costly framework are equivalent to scaled versions of solutions of corresponding traditional experiment design problems. This implies that it is possible to make use of computationally efficient algorithms developed for one framework in the other framework. In particular, during the last few years, very efficient LMI formulations have been developed independently for both frameworks (Bombois et al., 2006; Jansson, 2004; Jansson and Hjalmarsson, 2005a,b). The results presented in this paper can be used to translate these formulations between the two different approaches.
Additionally, the equivalence results allow the incorporation of additional constraints into the least costly framework. It also allows the results obtained in one framework to be interpreted in the other. For example, there are usually hard constraints on the input power or amplitude, due to actuator limitations. Thus, if the power of the optimal least costly input (where the cost has been measured in terms of the input power) exceeds the maximum allowed value, the equivalence results show that this is due to an excessively tight constraint on the model quality. Thus, by translating the problem into the traditional framework and then reverting back to the least costly framework, it is possible to modify this constraint in an appropriate way, in order to satisfy the hard input power constraint.

The remainder of the paper is organised as follows. In Section 2 we establish some preliminary results which will be used to show the equivalence between the various experiment design frameworks. In Section 3 we summarise notation and definitions from the least costly approach. In Section 4 we deal with the least costly framework for open loop design. In Section 5, we consider the closed loop case. Section 6 shows a simple example to illustrate the practical implications of the equivalence between the frameworks. Finally, Section 7 presents conclusions.

\section{Technical preliminaries}

Here we establish several preliminary results which will be utilised in the sequel to establish the equivalence between least costly and traditional experiment design problems for control. Theorem 3 below provides a duality result between two optimisation problems, where the roles of the cost function and constraints are exchanged. This result will be used repeatedly in the sequel to show the equivalence between the two experiment design frameworks.

Let $X$ be a cone ${ }^{1}$, and $f, g: X \rightarrow \mathbb{R}_{0}^{+}$be ${ }^{2}$ such that for every $x \in X$ and $\alpha>0, f(\alpha x)=\alpha f(x)$ and $g(\alpha x)=$ $\alpha^{-1} g(x)$.

Now define the following problems ${ }^{3}$ :

$$
\begin{aligned}
& \text { Problem } A: \min _{x \in X} f(x) \text { s.t. } g(x) \leq 1 . \\
& \text { Problem } B: \min _{y \in X} g(y) \text { s.t. } f(y) \leq 1 .
\end{aligned}
$$

Lemma 1 Assume that Problem A has a solution $x^{*} \in X$. Then $x^{*}$ is also a solution of the following problem:

$$
\text { Problem } A^{\prime}: \min _{x \in X} f(x) \text { s.t. } g(x)=1 \text {. }
$$

\footnotetext{
1 A set $X$ is a cone if it is subset of a vector space, and $x \in X$ implies that $\tau x \in X$ for all $\tau \geq 0$ (Rockafellar, 1970, p. 30).

$2 f$ and $g$ are called positively homogeneous functions of degree

1 and -1 , respectively (Lasserre and Hiriart-Urruty, 2002).

3 Here, 's.t.' denotes 'subject to'.
} 
PROOF. If $g\left(x^{*}\right)<1$, take $x=g\left(x^{*}\right) x^{*}$. Then, $g(x)=$ $\left[1 / g\left(x^{*}\right)\right] g\left(x^{*}\right)=1$ and $f(x)=g\left(x^{*}\right) f\left(x^{*}\right)<f\left(x^{*}\right)$. This contradicts the optimality of $x^{*}$. Thus, $g\left(x^{*}\right)=1$.

Corollary 2 Problem B is equivalent to

Problem $B^{\prime}: \min _{y \in X} g(y)$ s.t. $f(y)=1$.

PROOF. Follows from proof of Lemma 1.

Theorem 3 Problem A has a solution, $x^{*} \in X$, if and only if Problem $B$ has a solution, $y^{*} \in X$. Moreover, if a solution $x^{*}$ of Problem $A$ exists, then $x^{*}=K y^{*}$, where $K=f\left(x^{*}\right)=$ $g\left(y^{*}\right)$ and $y^{*}$ is a solution of Problem $B$, and vice versa.

PROOF. Let $x^{*} \in X$ be a solution of Problem A. Then, by Lemma $1, g\left(x^{*}\right)=1$. Take $K=f\left(x^{*}\right)$. Then, $y^{\prime}=K^{-1} x^{*}$ satisfies

$$
f\left(y^{\prime}\right)=f\left(K^{-1} x^{*}\right)=K^{-1} f\left(x^{*}\right)=\left[1 / f\left(x^{*}\right)\right] f\left(x^{*}\right)=1,
$$$$
g\left(y^{\prime}\right)=g\left(K^{-1} x^{*}\right)=K g\left(x^{*}\right)=f\left(x^{*}\right) g\left(x^{*}\right)=f\left(x^{*}\right) \text {. }
$$

Now, $g\left(y^{\prime}\right) \geq \inf _{y \in X, f(y)=1} g(y)$. Let us assume that $y^{\prime}$ is not a solution of Problem B, i.e. that $g\left(y^{\prime}\right)>\inf _{y \in X, f(y)=1} g(y)$. Then there is a $y^{\prime \prime} \in X$ such that $g\left(y^{\prime \prime}\right)<g\left(y^{\prime}\right)$ and $f\left(y^{\prime \prime}\right)=$ 1. Thus, taking $K^{\prime}=g\left(y^{\prime \prime}\right)$ and $x^{\prime}=K^{\prime} y^{\prime \prime}$, we have, by (1), that

$$
\begin{aligned}
& g\left(x^{\prime}\right)=g\left(K^{\prime} y^{\prime \prime}\right)=\left(K^{\prime}\right)^{-1} g\left(y^{\prime \prime}\right)=\left[1 / g\left(y^{\prime \prime}\right)\right] g\left(y^{\prime \prime}\right)=1, \\
& f\left(x^{\prime}\right)=K^{\prime} f\left(y^{\prime \prime}\right)=g\left(y^{\prime \prime}\right) f\left(y^{\prime \prime}\right)=g\left(y^{\prime \prime}\right)<g\left(y^{\prime}\right)=f\left(x^{*}\right) .
\end{aligned}
$$

This contradicts the optimality of $x^{*}$. Hence $g\left(y^{\prime}\right)=$ $\inf _{y \in X, f(y)=1} g(y)$, thus showing that $y^{\prime}$ is a solution of Problem B.

The converse can be established analogously.

Remark 4 To the best of our knowledge, Theorem 3 has not been stated in its full generality in the literature. However, a particular instance of Corollary 2 has been used in Hildebrand and Gevers, 2003, p. 1594.

\section{Basic definitions in experiment design}

In this section, we provide some basic definitions related to experiment design. For the motivation behind these definitions, the reader is referred to (Bombois et al., 2004a,b, 2006).

Consider the true system given by

$$
y_{t}=G\left(z, \theta_{T}\right) u_{t}+H\left(z, \theta_{T}\right) e_{t}, \quad \theta_{T} \in \mathbb{R}^{n},
$$

where $\left\{u_{t}\right\}$ is a quasi-stationary input signal having spectrum $\Phi_{u}$, and $\left\{e_{t}\right\}$ is white noise of variance $\sigma^{2}$. Here $z$ is considered to be either the forward shift operator, or the $Z$ transform variable, depending on whether the expressions are in the time or frequency domain, respectively.

Consider also a locally identifiable model structure given by $\mathcal{M}:=\left\{(G(z, \theta), H(z, \theta)): \quad \theta \in D_{\mathcal{M}} \subseteq \mathbb{R}^{n}\right\}$ (Ljung, 1999, p. 113), which includes the true system $\left(G\left(z, \theta_{T}\right), H\left(z, \theta_{T}\right)\right)$. Here, $G(z, \theta)$ and $H(z, \theta)$ are rational transfer functions, where the number of poles of $G(z, \theta)$ is $n^{\prime} \in \mathbb{N}$ (called the model order), $H(z, \theta)$ is stable and minimum phase, and $H(\infty, \theta)=1$. Let ${ }^{4}$ $\theta=:\left[\theta_{G}^{T} \theta_{G H}^{T} \theta_{H}^{T}\right]^{T}$, where $\theta_{G}, \theta_{H}$ and $\theta_{G H}$ contain the parameters which are exclusively in $G$, exclusively in $H$, and common to both $G$ and $H$, respectively.

Typically in experiment design, the optimal experiment depends on the true system, which is a priori unknown. In the least costly framework, an initial experiment is required. Therefore, in the sequel we assume that $\theta_{0}$ is an initial estimate of the true parameter vector $\theta_{T}$ (see e.g. (Rojas et al., 2007)), that has been obtained from a previous open-loop experiment using an input signal with spectrum $\Phi_{u, \text { init }}(\omega)$.

\subsection{Open loop experiment design}

Utilising a Prediction Error Method (PEM) to estimate the parameters in $\theta$, based on $N$ observations, it has been shown that, under mild conditions (Ljung, 1999, p. 282),

$$
\sqrt{N}\left(\hat{\theta}_{N}-\theta_{0}\right) \stackrel{d}{\rightarrow} \mathrm{N}\left(0, \bar{P}_{\theta_{0}}\right)
$$

where $\hat{\theta}_{N}$ is the PEM estimator of $\theta$, and

$$
\begin{aligned}
& \bar{P}_{\theta_{0}}^{-1}:=\frac{1}{2 \pi \sigma^{2}} \int_{-\pi}^{\pi} F_{u}\left(e^{j \omega}, \theta_{0}\right) F_{u}^{H}\left(e^{j \omega}, \theta_{0}\right) \Phi_{u}(\omega) d \omega \\
& +\frac{1}{2 \pi} \int_{-\pi}^{\pi} F_{e}\left(e^{j \omega}, \theta_{0}\right) F_{e}^{H}\left(e^{j \omega}, \theta_{0}\right) d \omega \\
& F_{u}(z, \theta):=H^{-1}(z, \theta) \Lambda_{G}(z, \theta) \\
& =\left[\begin{array}{lll}
F_{u, G}^{T}(z, \theta) & F_{u, G H}^{T}(z, \theta) & 0
\end{array}\right]^{T} \\
& F_{e}(z, \theta):=H^{-1}(z, \theta) \Lambda_{H}(z, \theta) \\
& =\left[0 F_{e, G H}^{T}(z, \theta) F_{e, H}^{T}(z, \theta)\right]^{T} \\
& \Lambda_{G}(z, \theta):=\frac{\partial G(z, \theta)}{\partial \theta} \\
& =\left[\Lambda_{G, G}^{T}(z, \theta) \Lambda_{G, G H}^{T}(z, \theta) 0\right]^{T} \\
& \Lambda_{H}(z, \theta):=\frac{\partial H(z, \theta)}{\partial \theta} \\
& =\left[\begin{array}{ll}
0 & \Lambda_{H, G H}^{T}(z, \theta) \Lambda_{H, H}^{T}(z, \theta)
\end{array}\right]^{T} .
\end{aligned}
$$

$\overline{4}$ If $A$ is a matrix, then $A^{T}$ and $A^{H}$ denote its transpose and conjugate transpose, respectively. 
Also note that $F_{u}, F_{e}, \Lambda_{G}$ and $\Lambda_{H}$ have been partitioned with respect to the parameters of $\theta=\left[\begin{array}{lll}\theta_{G}^{T} & \theta_{G H}^{T} & \theta_{H}^{T}\end{array}\right]^{T}$.

Thus, the variance of $\theta, P_{\theta_{0}}$, for large $N$, is asymptotically given by

$$
\begin{aligned}
P_{\theta_{0}}^{-1} \approx & N \bar{P}_{\theta_{0}}^{-1} \\
= & \frac{N}{2 \pi \sigma^{2}} \int_{-\pi}^{\pi} F_{u}\left(e^{j \omega}, \theta_{0}\right) F_{u}^{H}\left(e^{j \omega}, \theta_{0}\right) \Phi_{u}(\omega) d \omega \\
& \quad+\frac{N}{2 \pi} \int_{-\pi}^{\pi} F_{e}\left(e^{j \omega}, \theta_{0}\right) F_{e}^{H}\left(e^{j \omega}, \theta_{0}\right) d \omega .
\end{aligned}
$$

\subsection{Closed loop experiment design}

For a system operating under linear feedback, the input is generated by

$$
u_{t}=C(z)\left[r_{t}-y_{t}\right]
$$

where $C$ denotes a controller transfer function and $\left\{r_{t}\right\}$ is a quasi-stationary reference signal. In this case, under mild conditions, the asymptotic covariance of the PEM estimator of $\theta$ based on $N$ observations, $P_{\theta_{0}}$, satisfies (Ljung, 1999, p. 282)

$$
P_{\theta_{0}}^{-1}:=N P_{r}^{-1}+N P_{v}^{-1},
$$

where $N \in \mathbb{N}$ is the length of the experiment, $P_{v}^{-1} \in \mathbb{R}^{n \times n}$ is a fixed positive semi-definite symmetric matrix related to the influence of noise on $P_{\theta_{0}}$,

$$
P_{r}^{-1}:=\frac{1}{2 \pi \sigma^{2}} \int_{-\pi}^{\pi} F_{r}\left(e^{j \omega}, \theta_{0}\right) F_{r}^{H}\left(e^{j \omega}, \theta_{0}\right) \Phi_{r}(\omega) d \omega
$$

is the part of $P_{\theta_{0}}$ due to the reference signal, $\Phi_{r}$ is the reference spectrum,

$F_{r}\left(z, \theta_{0}\right):=H^{-1}\left(z, \theta_{0}\right) S_{i d}(z) \Lambda_{G}\left(z, \theta_{0}\right)$,

and $S_{i d}$ is an a-priori estimate of the loop sensitivity applicable during the experiment.

\subsection{Model confidence regions}

By utilising the covariance $P_{\theta_{0}}$, one is able to obtain the following confidence region of possible plant models (Bombois et al., 2001):

$$
\begin{aligned}
\mathcal{D}:=\{G(z, \theta) & =\frac{Z_{N}(z) \theta}{1+Z_{D}(z) \theta}: \\
& {\left.\left[\theta-\theta_{0}\right]^{T} P_{\theta_{0}}^{-1}\left[\theta-\theta_{0}\right]<\chi, \theta \in D_{\mathcal{M}}\right\}, }
\end{aligned}
$$

where $Z_{N}$ and $Z_{D}$ are row-vector-valued transfer functions related to $\mathcal{M}$, and $\chi>0$ is related to the confidence level of $\mathcal{D}$.
Now, define

$$
r_{u}(\omega):=\sqrt{\chi \hat{\lambda}\left\{T\left(e^{j \omega}, \theta_{0}\right) P_{\theta_{0}} T\left(e^{j \omega}, \theta_{0}\right)^{T}\right\}},
$$

where $^{5} T(z, \theta):=\left[\operatorname{Re} \Lambda_{G}(z, \theta) \operatorname{Im} \Lambda_{G}(z, \theta)\right]^{T}$. Notice that $T\left(e^{j \omega}, \theta_{0}\right) P_{\theta_{0}} T\left(e^{j \omega}, \theta_{0}\right)^{T}$ is positive semidefinite. Hence $r_{u}$ is a measure of the size of $\mathcal{D}$ (Bombois et al., 2004a), since ${ }^{6}$

$$
\begin{aligned}
& G \in \mathcal{D} \Rightarrow\left|G\left(e^{j \omega}, \theta\right)-G\left(e^{j \omega}, \theta_{0}\right)\right|<r_{u}(\omega), \\
& \forall \omega \in[-\pi, \pi], \theta \in D_{\mathcal{M}} .
\end{aligned}
$$

Let $r_{\text {adm }}:[-\pi, \pi] \rightarrow \mathbb{R}^{+}$be the largest admissible size of this region to guarantee a minimum level of closed-loop performance, based on an initial plant estimate (and a preselected fixed control design method, to be used with the model which will be obtained in the estimation stage to design a controller). See (Bombois et al., 2004a,b) for details on how $r_{\text {adm }}$ can be computed from an estimate of the controller that will be designed with the model to be obtained from the estimation stage. From the initial experiment, let $r_{\Phi, \text { init }}(\omega)$ be an estimate of the size of $\mathcal{D}$ obtained using the input spectrum $\Phi_{u, \text { init }}(\omega)$.

\subsection{Input/output power}

In the least costly approach to experiment design, the following cost function is considered:

$$
\begin{aligned}
\mathcal{J}_{r}:=\frac{1}{2 \pi} \int_{-\pi}^{\pi} & \left(\alpha_{u}\left|S_{i d}\left(e^{j \omega}\right)\right|^{2}\right. \\
& \left.+\alpha_{y}\left|G\left(e^{j \omega}, \theta_{0}\right) S_{i d}\left(e^{j \omega}\right)\right|^{2}\right) \Phi_{r}(\omega) d \omega
\end{aligned}
$$

where $\mathcal{J}_{r}$ is a weighted sum of the input and output power, with $\alpha_{u}$ and $\alpha_{y}$ the corresponding weights. This represents the power of the perturbations induced by the excitation signal $(u(t)$ or $r(t)$, depending on whether the identification is done in open or closed loop) on the normal operation signals.

The input power is given by the first term in the expression for $\mathcal{J}_{r}$. The output power is given by the second term, and is determined by $S_{i d}, \Phi_{r}$ and the initial estimate of the plant, $G\left(e^{j \omega}, \theta_{0}\right)$. In open loop experiment design, we take $\alpha_{u}=1$ and $\alpha_{y}=0$, i.e. we focus on the problem of minimising the input power.

\footnotetext{
$5 \hat{\lambda}\{A\}$ denotes the largest eigenvalue, or spectral radius, of a positive semi-definite matrix $A$ (Bernstein, 2005, p. 137).

6 Expression (6) is in fact valid only asymptotically as $N \rightarrow \infty$, since it is based on a Taylor approximation of the model $G(z, \theta)$ around $\theta=\theta_{0}$
} 


\subsection{Model quality}

In order to relate experiment design to robust control, model quality is defined in terms of the following functional

$$
J(G):=\left\|W_{l}\left[\begin{array}{cc}
\frac{G C}{1+G C} & \frac{G}{1+G C} \\
\frac{C}{1+G C} & \frac{1}{1+G C}
\end{array}\right] W_{r}\right\|_{\infty}
$$

where $J$ is an $\mathcal{H}_{\infty}$ performance measure determined by the plant, $G$, the controller, $C$, and the diagonal frequencydependent weighting transfer matrices $W_{l}$ and $W_{r}$. This definition will be considered only for the closed loop experiment design case. It should be noted that it could also be used in the open loop case.

\subsection{Signal spaces}

In the sequel we work with three spaces of input (or reference) spectra as defined below:

(1) Consider $\mathcal{U}_{1}$ as the space of all input spectra $\Phi_{u}$ which are uniformly bounded from above and below (i.e., such that $C \geq \Phi_{u}(\omega) \geq \delta>0$ for some $C$ and $\delta$ ). This requirement is necessary in order to apply the asymptotic covariance formula (Yuan and Ljung, 1984).

(2) Let $\mathcal{U}_{2}$ be the space of all input spectra $\Phi_{u}$ on $[-\pi, \pi]$ for which the open loop information matrix $P_{\theta_{0}}^{-1} \mathrm{de}$ fined by the right hand side of (3) is nonsingular and

$$
\begin{aligned}
\frac{N}{2 \pi \sigma^{2}} \int_{-\pi}^{\pi} \frac{\partial G\left(e^{j \omega}, \theta_{0}\right)}{\partial \rho} & {\left[\frac{\partial G\left(e^{j \omega}, \theta_{0}\right)}{\partial \rho}\right]^{H} } \\
& \cdot \frac{\Phi_{u}(\omega)}{\left|H\left(e^{j \omega}, \theta_{0}\right)\right|^{2}} d \omega>0,
\end{aligned}
$$

where $\rho:=\left[\begin{array}{ll}\theta_{G}^{T} & \theta_{G H}^{T}\end{array}\right]^{T}$. These conditions are equivalent to requiring that the system $\left(G\left(z, \theta_{0}\right), H\left(z, \theta_{0}\right)\right)$ is parameter identifiable under $\mathcal{M}$ and $\Phi_{u}$ for the maximum likelihood method (Söderström and Stoica, 1989, section 6.4), and also that the parameters of $G\left(\theta_{G}\right.$ and $\left.\theta_{G H}\right)$ should be identifiable for zero noise (i.e. $\left(G\left(z, \theta_{0}\right), 0\right)$ should be parameter identifiable under the modified model structure $\tilde{\mathcal{M}}:=\left\{(G(z, \theta), 0): \theta \in D_{\mathcal{M}}\right\}$ and $\Phi_{u}$, for the maximum likelihood method). Notice that these conditions impose restrictions on both the model structure and the input signal.

For model structures $\mathcal{M}$ such that $G$ and $H$ are independently parameterised, (7) is implied by the condition that the open-loop information matrix $P_{\theta_{0}}^{-1}$ is nonsingular, since then $P_{\theta_{0}}$ is block diagonal and its upper block is the inverse of the integral that appears in (7); see e.g. (Rojas et al., 2007).

In the case of certain standard model structures where $G$ and $H$ have parameters in common, condition
(7) usually reduces to requiring $u_{t}$ to be persistently exciting of a sufficiently high order (Ljung, 1999, section 13.2). For example, for an ARMAX structure having polynomial orders $n_{a}, n_{b}$ and $n_{c}, u_{t}$ should be persistently exciting of order $n_{a}+n_{b}$.

(3) Define $\mathcal{U}_{3}$ as the set of all reference spectra $\Phi_{r}$ on $[-\pi, \pi]$ for which the closed-loop information matrix $P_{\theta_{0}}^{-1}$ defined by the right side of (4) is nonsingular.

Spaces $\mathcal{U}_{1}$ and $\mathcal{U}_{2}$ will be considered for the case of openloop experiment design (i.e. when $\left\{u_{t}\right\}$ and $\left\{e_{t}\right\}$ are uncorrelated), and $\mathcal{U}_{3}$ will be used for the case of closed-loop experiment design.

Remark 5 It is well known (see e.g. Wold's Theorem (Priestley, 1981, Section 4.8.3)) that a generalised function (Lighthill, 1959) on $[-\pi, \pi]$ is the spectrum of a stationary stochastic process if and only if its antiderivative $F$ is an ordinary non-decreasing function. From this fact it can be readily seen that $\mathcal{U}_{1}, \mathcal{U}_{2}$ and $\mathcal{U}_{3}$ are cones, but not linear spaces (because multiplication by negative scalars is not allowed). This observation will be utilised in later sections where Theorem 3 will be applied to establish the equivalence between the least costly and traditional experiment design approaches.

Remark 6 For computational reasons, the spectra space is typically approximated by a subset of a finite dimensional space. With this approximation, the results presented in the sequel are equally valid, since the parametrisations are usually cones, as in the case of the 'finite dimensional spectrum' and 'partial correlation' parametrisations (Jansson and Hjalmarsson, 2005a). Moreover, condition (7) is automatically satisfied when the input spectrum is parameterised by a finite linear combination of rational basis functions, since the corresponding spectra are nonzero for almost all $\omega \in[0, \pi]$, i.e. persistently exciting of infinite order (Ljung, 1999, definition 13.2).

\section{Cheapest open-loop experiment design for control}

In this Section we consider the case when the system is operating in open loop. Three specific least costly problems are examined and are shown to be equivalent to traditional experiment design problems.

\subsection{High-order model approximation approach}

In this approach (Bombois et al., 2004b), the modeling error is assumed to be due to variance only and is hence approximated by the following asymptotic (in model order) covariance expression (Ljung, 1999, p. 294):

$$
P_{G}\left(e^{j \omega}\right):=\operatorname{Cov} G\left(e^{j \omega}, \hat{\theta}_{N}\right) \approx \frac{n^{\prime}}{N} \frac{\sigma^{2}\left|H\left(e^{j \omega}, \theta_{0}\right)\right|^{2}}{\Phi_{u}(\omega)} .
$$


where $n^{\prime}$ is the model order, as defined in Section 3. Notice that the covariance is asymptotically inversely proportional to $\Phi_{u}$. From this observation, it is possible to derive an expression for the covariance based on the data obtained from an initial experiment, i.e. $r_{\Phi, \text { init }}$ and $\Phi_{u, \text { init }}$. This is considered in (Bombois et al., 2004b), where it is noted that

$r_{u}^{2}(\omega) \approx r_{\Phi, \text { init }}^{2}(\omega) \frac{\Phi_{u, i n i t}(\omega)}{\Phi_{u}(\omega)}$

from which the model quality constraint " $r_{u}(\omega) \leq r_{\text {adm }}(\omega)$ for all $\omega \in[-\pi, \pi]$ " gives rise to the following problem:

Cheapest open-loop experiment design for control (based on a high-order model approximation):

$$
\begin{aligned}
& \min _{\Phi_{u} \in \mathcal{U}_{1}} \frac{1}{2 \pi} \int_{-\pi}^{\pi} \Phi_{u}(\omega) d \omega \\
& \text { s.t. } r_{\Phi, \text { init }}^{2}(\omega) \frac{\Phi_{u, \text { init }}(\omega)}{\Phi_{u}(\omega)} \leq r_{\text {adm }}^{2}(\omega), \forall \omega \in[-\pi, \pi] .
\end{aligned}
$$

where the definitions of $\mathcal{U}_{1}, \Phi_{u}, \Phi_{u, \text { init }}, r_{\Phi, \text { init }}$ and $r_{\text {adm }}$ are given in Section 3.

The equivalence between the above problem and a traditional experiment design problem is established in the following result:

Theorem 7 The cheapest open-loop experiment design for control (based on a high-order model approximation) problem is equivalent to the following traditional experiment design problem:

$$
\begin{aligned}
& \min _{\tilde{\Phi}_{u} \in \mathcal{U}_{1}}\left\|\frac{r_{\Phi, \text { init }}^{2}(\omega) \Phi_{u, \text { init }}(\omega)}{r_{\mathrm{adm}}^{2}(\omega) \tilde{\Phi}_{u}(\omega)}\right\|_{\infty} \\
& \text { s.t. } \frac{1}{2 \pi} \int_{-\pi}^{\pi} \tilde{\Phi}_{u}(\omega) d \omega \leq 1,
\end{aligned}
$$

in ${ }^{7}$ the sense that the solutions $\Phi_{u}^{*}$ and $\tilde{\Phi}_{u}^{*}$, if they exist, are related by $\Phi_{u}^{*}(\omega)=K \tilde{\Phi}_{u}^{*}(\omega)$ for every $\omega \in[-\pi, \pi]$, where $K:=(2 \pi)^{-1} \int_{-\pi}^{\pi} \Phi_{u}^{*}(\omega) d \omega$.

\footnotetext{
$\overline{7 \text { If } A:}[-\pi, \pi] \rightarrow \mathbb{C}^{n \times n}$ is (essentially) bounded on $\mathbb{R}$, then $\|A\|_{\infty}:=\operatorname{ess} \sup _{\omega \in[-\pi, \pi]} \bar{\sigma}[A(\omega)]$, where $\bar{\sigma}[A(\omega)]$ denotes the largest singular value of $A(\omega)$, and ess sup is the essential supremum; see e.g. (Zhou et al., 1996, p. 99).
}

PROOF. We define $f, g: \mathcal{U}_{1} \rightarrow \mathbb{R}_{0}^{+}$as

$$
\begin{aligned}
f\left(\Phi_{u}\right) & =\frac{1}{2 \pi} \int_{-\pi}^{\pi} \Phi_{u}(\omega) d \omega \\
g\left(\Phi_{u}\right) & =\sup _{\omega \in[-\pi, \pi]} \frac{r_{\Phi, \text { init }}^{2}(\omega) \Phi_{u, \text { init }}(\omega)}{r_{\mathrm{adm}}^{2}(\omega) \Phi_{u}(\omega)} \\
& =\left\|\frac{r_{\Phi, \text { init }}^{2}(\omega) \Phi_{u, \text { init }}(\omega)}{r_{\mathrm{adm}}^{2}(\omega) \Phi_{u}(\omega)}\right\|_{\infty} .
\end{aligned}
$$

Then application of Theorem 3 immediately provides the result, since for every $\Phi_{u} \in \mathcal{U}_{1}$ and $\alpha>0, f\left(\alpha \Phi_{u}\right)=$ $\alpha f\left(\Phi_{u}\right)$, and $g\left(\alpha \Phi_{u}\right)=\alpha^{-1} g\left(\Phi_{u}\right)$.

Remark 8 Notice that the equivalent traditional problem can be stated in terms of the covariance of $G, P_{G}$, as:

$$
\begin{aligned}
& \min _{\tilde{\Phi}_{u} \in \mathcal{U}_{1}}\left\|\frac{1}{r_{\mathrm{adm}}^{2}(\omega)} P_{G}\left(e^{j \omega}\right)\right\|_{\infty} \\
& \text { s.t. } \frac{1}{2 \pi} \int_{-\pi}^{\pi} \tilde{\Phi}_{u}(\omega) d \omega \leq 1 .
\end{aligned}
$$

This is because $r_{\Phi, \text { init }}^{2}(\omega) \Phi_{u, \text { init }}(\omega) / \Phi_{u}(\omega)$ is proportional to $P_{G}\left(e^{j \omega}\right)$, according to (8). This last problem reflects the traditional purpose of designing an input signal to minimise some scalar function of the covariance of $G$ or $\theta$, subject to input and/or output power constraints.

\subsection{Finite-order model approach}

Here we consider the use of covariance expressions based on a finite model order (Ljung, 1999, Section 9.4). In this case, the experiment design problem can be stated in the least costly framework (Bombois et al., 2004a) as

\section{Cheapest Experiment Design Problem for Control:}

$$
\begin{aligned}
& \min _{\Phi_{u} \in \mathcal{U}_{2}} \frac{1}{2 \pi} \int_{-\pi}^{\pi} \Phi_{u}(\omega) d \omega \\
& \text { s.t. } r_{u}(\omega) \leq r_{\mathrm{adm}}(\omega), \quad \forall \omega \in[-\pi, \pi],
\end{aligned}
$$

where the definitions of $\mathcal{U}_{2}, \Phi_{u}, r_{u}$ and $r_{\text {adm }}$ are given in Section 3.

In this problem, it can be seen that one seeks to minimise the input power subject to a measure of the model uncertainty being less than a control based performance constraint. The equivalence between this particular cheapest experiment design problem and traditional open-loop experiment design is established in the following result:

Theorem 9 If the model structure $\mathcal{M}$ is such that $G$ and $H$ are independently parameterised (i.e. for Box-Jenkins, Output-Error and FIR models), then the cheapest experiment design problem for control is equivalent to the following 
problem:

$$
\begin{aligned}
& \min _{\tilde{\Phi}_{u} \in \mathcal{U}_{2}}\left\|\frac{1}{r_{\mathrm{adm}}^{2}(\omega)} T\left(e^{j \omega}, \theta_{0}\right) P_{\theta_{0}} T^{T}\left(e^{j \omega}, \theta_{0}\right)\right\|_{\infty} \\
& \text { s.t. } \frac{1}{2 \pi} \int_{-\pi}^{\pi} \tilde{\Phi}_{u}(\omega) d \omega \leq 1,
\end{aligned}
$$

in the sense that the solutions $\Phi_{u}^{*}$ and $\tilde{\Phi}_{u}^{*}$, if they exist, are related by $\Phi_{u}^{*}(\omega)=K \tilde{\Phi}_{u}^{*}(\omega)$ for every $\omega \in[-\pi, \pi]$, where $K:=(2 \pi)^{-1} \int_{-\pi}^{\pi} \Phi_{u}^{*}(\omega) d \omega$.

PROOF. As in the proof of Theorem 7, we define

$$
\begin{aligned}
f\left(\Phi_{u}\right) & :=\frac{1}{2 \pi} \int_{-\pi}^{\pi} \Phi_{u}(\omega) d \omega, \\
g\left(\Phi_{u}\right) & :=\sup _{\omega \in[-\pi, \pi]} \frac{1}{r_{\mathrm{adm}}^{2}(\omega)} r_{u}^{2}(\omega),
\end{aligned}
$$

Notice that, by the definition of $r_{u}$ in (5),

$$
\begin{aligned}
& r_{u}^{2}(\omega) \\
& =\chi \hat{\lambda}\left\{T\left(e^{j \omega}, \theta_{0}\right) P_{\theta_{0}} T^{T}\left(e^{j \omega}, \theta_{0}\right)\right\} \\
& =\frac{2 \pi \chi \sigma^{2}}{N} \hat{\lambda}\left\{T\left(e^{j \omega}, \theta_{0}\right)\right. \\
& \cdot\left[\int_{-\pi}^{\pi} F_{u}\left(e^{j \tau}, \theta_{0}\right) F_{u}^{H}\left(e^{j \tau}, \theta_{0}\right) \Phi_{u}(\tau) d \tau\right. \\
& \left.+\sigma^{2} \int_{-\pi}^{\pi} F_{e}\left(e^{j \tau}, \theta_{0}\right) F_{e}^{H}\left(e^{j \tau}, \theta_{0}\right) d \tau\right]^{-1} \\
& \left.\cdot T^{T}\left(e^{j \omega}, \theta_{0}\right)\right\} \text {. }
\end{aligned}
$$

Given that $G$ and $H$ are independently parameterised, then, according to the partition $\theta=\left[\begin{array}{ll}\theta_{G}^{T} & \theta_{H}^{T}\end{array}\right]^{T}$,

$$
\begin{aligned}
& \int_{-\pi}^{\pi} F_{u}\left(e^{j \tau}, \theta_{0}\right) F_{u}^{H}\left(e^{j \tau}, \theta_{0}\right) \Phi_{u}(\tau) d \tau \\
& =\left[\frac{\int_{-\pi}^{\pi} F_{u, G}\left(e^{j \tau}, \theta_{0}\right) F_{u, G}^{T}\left(e^{-j \tau}, \theta_{0}\right) \Phi_{u}(\tau) d \tau \mid 0}{0}\right] \\
& \sigma^{2} \int_{-\pi}^{\pi} F_{e}\left(e^{j \tau}, \theta_{0}\right) F_{e}^{H}\left(e^{j \tau}, \theta_{0}\right) d \tau \\
& =\left[\frac{0}{0 \mid \sigma^{2} \int_{-\pi}^{\pi} F_{e, H}\left(e^{j \tau}, \theta_{0}\right) F_{e, H}^{T}\left(e^{-j \tau}, \theta_{0}\right) d \tau}\right] \\
& T^{T}\left(e^{j \omega}, \theta_{0}\right)=\left[\frac{\operatorname{Re} \Lambda_{G, G}\left(e^{j \omega}, \theta_{0}\right) \operatorname{Im} \Lambda_{G, G}\left(e^{j \omega}, \theta_{0}\right)}{0}\right]
\end{aligned}
$$

hence the sum of the integrals in (9) is a block-diagonal matrix, whose inverse is block-diagonal as well (Bernstein, 2005, fact 2.15.1). Then, pre- and post-multiplication by $T$ and $T^{T}$ respectively, shows that the cost function takes into account only the upper-left block of the sum, which is related to the integral of $F_{u} F_{u}^{T} \Phi_{u}$. Thus, $r_{u}$ can be written in terms of the Moore-Penrose generalised inverse, denoted $\dagger$, (Bernstein, 2005, chapter 6) as

$$
\begin{aligned}
& r_{u}^{2}(\omega) \\
& =\frac{2 \pi \chi \sigma^{2}}{N} \hat{\lambda}\left\{T\left(e^{j \omega}, \theta_{0}\right)\right. \\
& {\left[\begin{array}{c|c|c}
\left(\int_{-\pi}^{\pi} F_{u, G}\left(e^{j \tau}, \theta_{0}\right) F_{u, G}^{T}\left(e^{-j \tau}, \theta_{0}\right) \Phi_{u}(\tau) d \tau\right)^{-1} & 0 \\
\hline 0 & 0
\end{array}\right]} \\
& \left.\cdot T^{T}\left(e^{j \omega}, \theta_{0}\right)\right\} \\
& =\frac{2 \pi \chi \sigma^{2}}{N} \hat{\lambda}\left\{T\left(e^{j \omega}, \theta_{0}\right)\right. \\
& \cdot\left[\int_{-\pi}^{\pi} F_{u}\left(e^{j \tau}, \theta_{0}\right) F_{u}^{H}\left(e^{j \tau}, \theta_{0}\right) \Phi_{u}(\tau) d \tau\right]^{\dagger} \\
& \left.\cdot T^{T}\left(e^{j \omega}, \theta_{0}\right)\right\} \text {. }
\end{aligned}
$$

Therefore $\left.r_{u}^{2}(\omega)\right|_{\alpha \Phi_{u}}=\left.\alpha^{-1} r_{u}^{2}(\omega)\right|_{\Phi_{u}}$ for every $\omega \in$ $[-\pi, \pi]$, and $g\left(\alpha \Phi_{u}\right)=\alpha^{-1} g\left(\Phi_{u}\right)$. We can now proceed as in the proof of Theorem 7, since $g$ can be written in terms of the infinity norm, and the constant $\chi$ is irrelevant.

In the case of model structures $\mathcal{M}$ such that $G$ and $H$ are not independently parameterised, it is more difficult, but still possible to find a traditional equivalent of the cheapest experiment design problem for control. To this end, we introduce the following definition:

Definition 10 Let $A \in \mathbb{R}^{n \times n}$ be symmetric. Then, if $V \in$ $\mathbb{R}^{n \times n}$ such that $A=V^{T} D V$, where $D \in \mathbb{R}^{n \times n}$ is diagonal (Bernstein, 2005, fact 5.8.16), we define $[A]_{+}$as a Cholesky Factor of $(1 / 2) V^{T}(D+|D|) V$, i.e. ${ }^{8}$,

$$
[A]_{+}^{T}[A]_{+}=\frac{1}{2} V^{T}(D+|D|) V .
$$

Note that $[A]_{+}$is not uniquely defined in Definition 10 . This is not an issue in the following results, as it is not a requirement for the equivalence to be unique. The following result establishes the equivalence between the cheapest experiment design and traditional methodologies in the present case:

\footnotetext{
${ }_{8}|D|$ is the absolute value of a matrix $D$ (Bernstein, 2005, p. 343).
} 
Theorem 11 Consider a model structure, $\mathcal{M}$, such that $G$ and $H$ are not necessarily independently parameterised (i.e. for ARX and ARMAX models). In this case the cheapest experiment design problem for control is equivalent to the following problem:

$$
\begin{aligned}
& \min _{\tilde{\Phi}_{u} \in \mathcal{U}_{2}}\left\|W\left(e^{j \omega}, \theta_{0}\right) P_{u} W^{T}\left(e^{j \omega}, \theta_{0}\right)\right\|_{\infty} \\
& \text { s.t. } \frac{1}{2 \pi} \int_{-\pi}^{\pi} \tilde{\Phi}_{u}(\omega) d \omega \leq 1,
\end{aligned}
$$

where

$$
\begin{gathered}
W\left(e^{j \omega}, \theta_{0}\right):=\left[\frac{\chi}{r_{a d m}^{2}(\omega)} T^{T}\left(e^{j \omega}, \theta_{0}\right) T\left(e^{j \omega}, \theta_{0}\right)\right. \\
\left.-\frac{N}{2 \pi} \int_{-\pi}^{\pi} F_{e}\left(e^{j \tau}, \theta_{0}\right) F_{e}^{H}\left(e^{j \tau}, \theta_{0}\right) d \tau\right]_{+} \\
P_{u}:=\left[\frac{N}{2 \pi \sigma^{2}} \int_{-\pi}^{\pi} F_{u}\left(e^{j \tau}, \theta_{0}\right) F_{u}^{H}\left(e^{j \tau}, \theta_{0}\right) \Phi_{u}(\tau) d \tau\right]^{\dagger} .
\end{gathered}
$$

The equivalence holds in the sense that the solutions $\Phi_{u}^{*}$ and $\tilde{\Phi}_{u}^{*}$, if they exist, are related by $\Phi_{u}^{*}(\omega)=K \tilde{\Phi}_{u}^{*}(\omega)$ for every $\omega \in[-\pi, \pi]$, where $K:=(2 \pi)^{-1} \int_{-\pi}^{\pi} \Phi_{u}^{*}(\omega) d \omega$.

PROOF. Notice that, for each $\omega \in[-\pi, \pi]$, the definition of $r_{u}$ in (5) implies

$$
\begin{aligned}
& r_{u}(\omega) \leq r_{\mathrm{adm}}(\omega) \\
& \quad \Leftrightarrow \quad \chi \hat{\lambda}\left\{T\left(e^{j \omega}, \theta_{0}\right) P_{\theta_{0}} T^{T}\left(e^{j \omega}, \theta_{0}\right)\right\} \leq r_{\mathrm{adm}}^{2}(\omega) \\
& \quad \Leftrightarrow \quad \frac{r_{\mathrm{adm}}^{2}(\omega)}{\chi} I-T\left(e^{j \omega}, \theta_{0}\right) P_{\theta_{0}} T^{T}\left(e^{j \omega}, \theta_{0}\right) \geq 0 \\
& \quad \Leftrightarrow \quad P_{\theta_{0}}^{-1}-\frac{\chi}{r_{\mathrm{adm}}^{2}(\omega)} T^{T}\left(e^{j \omega}, \theta_{0}\right) T\left(e^{j \omega}, \theta_{0}\right) \geq 0 .
\end{aligned}
$$

The equivalence between lines 2 and 3 is due to a characterization of positive definiteness in terms of the spectral radius (Bernstein, 2005, fact 8.15.4). Lines 4 and 5 are equivalent due to a property of Schur complements for nonstrict inequalities (Bernstein, 2005, proposition 8.2.3; Boyd et al., 1994, p. 28). Now, by (3) and (10), we have that

$$
P_{\theta_{0}}^{-1}=P_{u}^{\dagger}+\frac{N}{2 \pi} \int_{-\pi}^{\pi} F_{e}\left(e^{j \omega}, \theta_{0}\right) F_{e}^{H}\left(e^{j \omega}, \theta_{0}\right) d \omega
$$

Thus, we obtain

$$
\begin{array}{lc}
r_{u}(\omega) \leq r_{\mathrm{adm}}(\omega) \\
\Leftrightarrow \quad P_{u}^{\dagger} \geq \frac{\chi}{r_{\mathrm{adm}}^{2}(\omega)} T^{T}\left(e^{j \omega}, \theta_{0}\right) T\left(e^{j \omega}, \theta_{0}\right) \\
& -\frac{N}{2 \pi} \int_{-\pi}^{\pi} F_{e}\left(e^{j \tau}, \theta_{0}\right) F_{e}^{H}\left(e^{j \tau}, \theta_{0}\right) d \tau \\
\Leftrightarrow & P_{u}^{\dagger} \geq W^{T}\left(e^{j \omega}, \theta_{0}\right) W\left(e^{j \omega}, \theta_{0}\right) \\
\Leftrightarrow \quad & I-W\left(e^{j \omega}, \theta_{0}\right) P_{u}\left(\Phi_{u}\right) W^{T}\left(e^{j \omega}, \theta_{0}\right) \geq 0, \\
\Leftrightarrow & W\left(e^{j \omega}, \theta_{0}\right) P_{u} W^{T}\left(e^{j \omega}, \theta_{0}\right) \leq I, \\
& {\left[I-P_{u}^{\dagger} P_{u}\right] W^{T}\left(e^{j \omega}, \theta_{0}\right)=0} \\
\Leftrightarrow & \hat{\lambda}\left\{W\left(e^{j \omega}, \theta_{0}\right) P_{u} W^{T}\left(e^{j \omega}, \theta_{0}\right)\right\} \leq 1, \\
& {\left[I-P_{u}^{\dagger} P_{u}\right] W^{T}\left(e^{j \omega}, \theta_{0}\right)=0 .}
\end{array}
$$

Here, the equivalence between lines 2 and 3 follows from Lemma 16 of the Appendix, since $P_{u}^{\dagger} \geq 0$. Also, the equivalence between lines 3 and 4 is due to a property of Schur complements for nonstrict inequalities, and the equivalence between lines 5 and 6 comes from the characterization of positive definiteness in terms of the spectral radius.

By Lemma 17 of the Appendix, $\left[I-P_{u}^{\dagger} P_{u}\right] W^{T}\left(e^{j \omega}, \theta_{0}\right)=$ 0 . Since (11) holds for every $\omega \in[-\pi, \pi]$, then

$$
\begin{aligned}
& r_{u}(\omega) \leq r_{\mathrm{adm}}(\omega), \quad \forall \omega \in[-\pi, \pi] \\
& \quad \Leftrightarrow \quad \sup _{\omega \in[-\pi, \pi]} \hat{\lambda}\left\{W\left(e^{j \omega}, \theta_{0}\right) P_{u} W^{T}\left(e^{j \omega}, \theta_{0}\right)\right\} \leq 1 \\
& \quad \Leftrightarrow \quad\left\|W\left(e^{j \omega}, \theta_{0}\right) P_{u} W^{T}\left(e^{j \omega}, \theta_{0}\right)\right\|_{\infty} \leq 1 .
\end{aligned}
$$

Hence, taking

$$
\begin{aligned}
f\left(\Phi_{u}\right) & =\frac{1}{2 \pi} \int_{-\pi}^{\pi} \Phi_{u}(\omega) d \omega \\
g\left(\Phi_{u}\right) & =\left\|W\left(e^{j \omega}, \theta_{0}\right) P_{u} W^{T}\left(e^{j \omega}, \theta_{0}\right)\right\|_{\infty}
\end{aligned}
$$

the rest of the proof follows as in Theorem 7.

Theorems 9 and 11 explicitly show the equivalence between the least costly framework and traditional open loop experiment design problems for the most commonly used model structures.

\section{Least costly closed-loop experiment design for control}

Now we consider the case when the system is operating under closed loop control. In this setting, Bombois et al., 2005, 2006 considered the use of covariance expressions based on a finite model order (Ljung, 1999, Section 9.4). In this framework, the following experiment design problem 
has been stated:

Least Costly Identification Experiment for Control:

$\min _{\Phi_{r} \in \mathcal{U}_{3}} \mathcal{J}_{r}$

s.t. $J(G) \leq 1, \quad \forall G \in \mathcal{D}$.

where $\Phi_{r}, \mathcal{U}_{3}, \mathcal{J}_{r}, J(G)$ and $\mathcal{D}$ are as defined in Section 3.

The following result establishes the equivalence between the closed-loop least costly framework and traditional experiment design, based on covariance expressions related to the use of a finite model order:

Theorem 12 The least costly identification experiment for control problem is equivalent to the following problem $^{9}$ :

$$
\begin{aligned}
& \min _{\tilde{\Phi}_{r} \in \mathcal{U}_{3}\{\theta: J(G(\theta))>1\}} \sup _{\text {s.t. } \mathcal{J}_{r} \leq 1,} \frac{\chi / N-\left[\theta-\theta_{0}\right]^{T} P_{v}^{-1}\left[\theta-\theta_{0}\right]}{\left[\theta-\theta_{0}\right]^{T} P_{r}^{-1}\left[\theta-\theta_{0}\right]} \\
& \text { ( }
\end{aligned}
$$

(where $G(\theta):=Z_{N} \theta /\left(1+Z_{D} \theta\right)$ ) in the sense that the solutions $\Phi_{r}^{*}$ and $\tilde{\Phi}_{r}^{*}$, if they exist, are related by $\Phi_{r}^{*}(\omega)=K \tilde{\Phi}_{r}^{*}(\omega)$ for every $\omega \in[-\pi, \pi]$, where $K:=(2 \pi)^{-1} \int_{-\pi}^{\pi} \Phi_{r}^{*}(\omega) d \omega$.

PROOF. Notice that the condition " $J(G) \leq 1, \forall G \in \mathcal{D}$ " can be written as

$$
\begin{aligned}
& \{G \in \mathcal{D} \Rightarrow J(G) \leq 1\} \\
& \Leftrightarrow\{J(G)>1 \Rightarrow G \notin \mathcal{D}\} \\
& \Leftrightarrow\left\{J(G(\theta))>1 \Rightarrow\left[\theta-\theta_{0}\right]^{T} P_{\theta_{0}}^{-1}\left[\theta-\theta_{0}\right] \geq \chi\right\} \\
& \Leftrightarrow\left\{J(G(\theta))>1 \Rightarrow\left[\theta-\theta_{0}\right]^{T} P_{r}^{-1}\left[\theta-\theta_{0}\right]\right. \\
& \left.\quad \geq \chi / N-\left[\theta-\theta_{0}\right]^{T} P_{v}^{-1}\left[\theta-\theta_{0}\right]\right\} \\
& \Leftrightarrow\left\{J(G(\theta))>1 \Rightarrow \frac{\chi / N-\left[\theta-\theta_{0}\right]^{T} P_{v}^{-1}\left[\theta-\theta_{0}\right]}{\left[\theta-\theta_{0}\right]^{T} P_{r}^{-1}\left[\theta-\theta_{0}\right]} \leq 1\right\} \\
& \Leftrightarrow \sup _{\{\theta: J(G(\theta))>1\}} \frac{\chi / N-\left[\theta-\theta_{0}\right]^{T} P_{v}^{-1}\left[\theta-\theta_{0}\right]}{\left[\theta-\theta_{0}\right]^{T} P_{r}^{-1}\left[\theta-\theta_{0}\right]} \leq 1 .
\end{aligned}
$$

Thus, setting

$$
\begin{aligned}
& f\left(\Phi_{u}\right)=\mathcal{J}_{r}, \\
& g\left(\Phi_{u}\right)=\sup _{\{\theta: J(G(\theta))>1\}} \frac{\chi / N-\left[\theta-\theta_{0}\right]^{T} P_{v}^{-1}\left[\theta-\theta_{0}\right]}{\left[\theta-\theta_{0}\right]^{T} P_{r}^{-1}\left[\theta-\theta_{0}\right]}
\end{aligned}
$$

we can proceed as in the proof of Theorem 7.

\footnotetext{
${ }^{9}$ Here we adopt the convention that $a / 0=+\infty$ if $a>0$, $a / 0=-\infty$ if $a<0$, and $0 / 0=0$.
}

Corollary 13 The least costly identification experiment for control problem is also equivalent to the following problem $^{10}$ :

$$
\begin{aligned}
& \max _{\tilde{\Phi}_{r} \in \mathcal{U}_{3}\{\theta: J(G(\theta))>1\}} \inf _{\{t} \operatorname{tr}\left\{W(\theta) P_{\theta_{0}}^{-1}\right\}-g(\theta) \\
& \text { s.t. } \mathcal{J}_{r} \leq 1,
\end{aligned}
$$

where

$$
\begin{aligned}
W(\theta) & :=\frac{\left[\theta-\theta_{0}\right]\left[\theta-\theta_{0}\right]^{T}}{\chi-\left[\theta-\theta_{0}\right]^{T} N P_{v}^{-1}\left[\theta-\theta_{0}\right]} \\
g(\theta) & :=\frac{\chi}{\chi-\left[\theta-\theta_{0}\right]^{T} N P_{v}^{-1}\left[\theta-\theta_{0}\right]} .
\end{aligned}
$$

PROOF. This can be seen from Theorem 12, by inverting the cost function and noting that

$$
\begin{aligned}
& \frac{\left[\theta-\theta_{0}\right]^{T} P_{r}^{-1}\left[\theta-\theta_{0}\right]}{\chi / N-\left[\theta-\theta_{0}\right]^{T} P_{v}^{-1}\left[\theta-\theta_{0}\right]} \\
& =\frac{\left[\theta-\theta_{0}\right]^{T} N P_{r}^{-1}\left[\theta-\theta_{0}\right]}{\chi-\left[\theta-\theta_{0}\right]^{T} N P_{v}^{-1}\left[\theta-\theta_{0}\right]} \\
& =\frac{\left[\theta-\theta_{0}\right]^{T} P_{\theta_{0}}^{-1}\left[\theta-\theta_{0}\right]-\left[\theta-\theta_{0}\right]^{T} N P_{v}^{-1}\left[\theta-\theta_{0}\right]}{\chi-\left[\theta-\theta_{0}\right]^{T} N P_{v}^{-1}\left[\theta-\theta_{0}\right]} \\
& =\frac{\left[\theta-\theta_{0}\right]^{T} P_{\theta_{0}}^{-1}\left[\theta-\theta_{0}\right]}{\chi-\left[\theta-\theta_{0}\right]^{T} N P_{v}^{-1}\left[\theta-\theta_{0}\right]} \\
& -\frac{\left[\theta-\theta_{0}\right]^{T} N P_{v}^{-1}\left[\theta-\theta_{0}\right]}{\chi-\left[\theta-\theta_{0}\right]^{T} N P_{v}^{-1}\left[\theta-\theta_{0}\right]} \\
& =\operatorname{tr}\left\{\frac{\left[\theta-\theta_{0}\right]\left[\theta-\theta_{0}\right]^{T}}{\chi-\left[\theta-\theta_{0}\right]^{T} N P_{v}^{-1}\left[\theta-\theta_{0}\right]} P_{\theta_{0}}^{-1}\right\} \\
& +1-\frac{\chi}{\chi-\left[\theta-\theta_{0}\right]^{T} N P_{v}^{-1}\left[\theta-\theta_{0}\right]},
\end{aligned}
$$

where the last line follows from the fact that $\operatorname{tr} A B=\operatorname{tr} B A$ for any matrices $A, B$ such that $A B$ is square (Bernstein, 2005 , p. 22). The constant term 1 in (12) can be omitted from the optimisation problem.

Remark 14 The problem formulated in Corollary 13 is similar to a popular traditional experiment design problem described e.g. in Mehra, 1974, except for the inclusion of the term $-g$ and the minimisation with respect to $\theta$, which can be interpreted as a form of 'robustification' with respect to uncertainty regarding the prior knowledge of $\theta$ (Rojas et al., 2007).

\section{An Illustrative Example}

The results presented in the previous sections show that solutions of several least costly experiment design problems

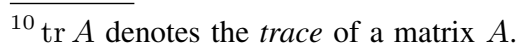


are scaled versions of solutions of corresponding traditional experiment design problems. This implies that it is possible to use computationally efficient algorithms developed for one framework in the other one.

Also, the equivalence results allow the incorporation and interpretation of additional constraints into the least costly framework. This is illustrated with a very simple example.

Example 15 Consider the problem of designing an input signal of minimum power to identify (in open loop) a FIR system of order $n$, so that the asymptotic variance of the transfer function estimator is uniformly bounded by a constant $1 /(N \gamma)$ in a given bandwidth $\left[-\omega_{B}, \omega_{B}\right]$, where $N$ is the data length. This is a least costly experiment design formulation, which can be written as the following optimization problem:

$$
\begin{array}{ll}
\min _{\Phi_{u}} & \frac{1}{2 \pi} \int_{-\pi}^{\pi} \Phi_{u}(\omega) d \omega \\
\text { s.t. } & \Phi_{u}(\omega) \geq 0, \quad|\omega| \leq \pi \\
& \lim _{N \rightarrow \infty} N \operatorname{Var}\left\{G\left(e^{j \omega}, \hat{\theta}_{N, n}\right)\right\} \leq \frac{1}{\gamma}, \quad|\omega| \leq \omega_{B} .
\end{array}
$$

where, by the Gauss' approximation formula (Ljung, 1999, page 292),

$$
\lim _{N \rightarrow \infty} N \operatorname{Var}\left\{G\left(e^{j \omega}, \hat{\theta}_{N, n}\right)\right\}=\sigma^{2} \Lambda_{n}^{H}\left(e^{j \omega}\right) T_{n}^{-1} \Lambda_{n}\left(e^{j \omega}\right) .
$$

which will be called the normalised variance of $G$. Here $T_{n}$ ia a Toeplitz matrix of the vector $\left[\begin{array}{llll}r_{0} & r_{1} & \cdots & r_{n}\end{array}\right]$ of the first $n+1$ lags of the autocovariance sequence of $u$, and $\Lambda_{n}(q):=\left[\begin{array}{llll}1 & q^{-1} & \cdots & q^{-n}\end{array}\right]^{T}$.

This problem has been studied, e.g., in (Rojas et al., 2008), where the techniques of (Jansson and Hjalmarsson, 2005a) have been used to formulate it as an semidefinite program. Notice that the optimal input is not unique, since the problem depends only on the first $n+1$ lags of the autocorrelation sequence of $u$.

Figure 1 shows the normalised variance of the frequency response obtained with the optimal inputs, designed for $n=$ $6, \sigma^{2}=\gamma=1$ and $\omega_{B}=0.8 \pi$. In this case, the minimum input power required is approximately 6.8674 .

Now, in practice there is always a constraint, say $P$, on the maximum input power. To analyse the effect of this constraint, notice that an equivalent traditional experiment design problem, according to Theorem 3, is

$$
\begin{array}{ll}
\min _{\Phi_{u}} & \max _{\omega \in\left[-\omega_{B}, \omega_{B}\right]} \gamma \lim _{N \rightarrow \infty} N \operatorname{Var}\left\{G\left(e^{j \omega}, \hat{\theta}_{N, n}\right)\right\} \\
\text { s.t. } \quad & \Phi_{u}(\omega) \geq 0, \quad|\omega| \leq \pi \\
& \frac{1}{2 \pi} \int_{-\pi}^{\pi} \Phi_{u}(\omega) d \omega \leq P .
\end{array}
$$

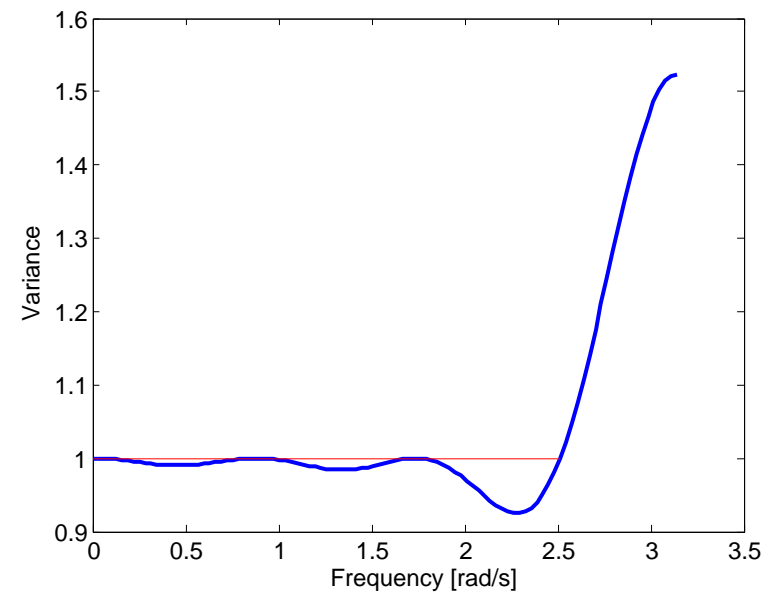

Fig. 1. Normalised variance of the frequency response of an FIR model of order 10, identified using an optimal input signal (thick). Required upper bound on the normalised variance (thin).

If $P>6.8674$, this means that there is available some extra input power which can be used to obtain an even better model, if possible. To this end, the duality between the least costly and traditional problems establishes that it suffices to scale the optimal inputs by $P / 6.8674>1$ to obtain an input for which the obtained model's quality is the best possible (in the sense described by the cost function of (13)) while taking advantage of the full input power available.

On the other hand, if $P<6.8674$, it means that there is less input power available than necessary to satisfy the model quality constraint. We then need to determine what is the best we can do now.

Again, from the equivalent traditional experiment design problem, it is readily seen that if $P<6.8674$, we can simply scale the optimal solutions of the least costly problem by $P / 6.8674<1$ to get inputs using the full power available to give the best possible model (according to the model quality criterion described by the cost function of (13)).

Notice that when there are hard constraints on both the input and output power, the results of this paper show that the traditional experiment design problem is actually equivalent to a minimax least costly problem. To see this in the open loop case, notice that the hard constraints on both the input and output power can be combined into a constraint of the form

$\max \left\{\int_{-\pi}^{\pi} \Phi_{u}(\omega) d \omega, \int_{-\pi}^{\pi}\left|G\left(e^{j \omega}, \theta_{0}\right)\right|^{2} \Phi_{u}(\omega) d \omega\right\} \leq 1$,

where $\Phi_{u}$ and $G$ have been properly scaled. The left hand side of this constraint is proportional to $\Phi_{u}$, so an equivalent least costly problem consists of minimising this quantity, subject to a model quality constraint. 


\section{Conclusions}

In this paper, we have shown, via a duality result, that least costly identification experiment design for control can be formulated in a more traditional setting, by stating it as an $\mathcal{H}_{\infty}$ or minimax optimisation problem, depending on the precise constraints being taken into account.

Specifically, we have considered 4 problems from the least costly framework. In the open loop case, we have established equivalence to the traditional experiment design problems using high order and finite order approximations of the covariance, for both independently and non-independently parameterised plant and noise models. For systems operating in closed loop, equivalence has been shown when using covariance expressions which are non asymptotic in the model order.

We believe that this duality between least costly and traditional experiment design gives new insights into both frameworks. It also offers practical advantages e.g. by allowing the different computational tools developed for each problem to be used in either framework. Finally, the results are believed to provide a better understanding of the link between identification and robust control.

\section{Appendix. Technical lemmas}

Lemma 16 Let $A, B \in \mathbb{R}^{n \times n}$ be symmetric, where $A \geq 0$. Then, it follows that $A \geq B$ if and only if $A \geq[B]_{+}^{T}[B]_{+}$.

PROOF. Let $V \in \mathbb{R}^{n \times n}$ be such that $B=V^{T} D V$, where $D \in \mathbb{R}^{n \times n}$ is diagonal (Bernstein, 2005, fact 5.8.16). Then, $A \geq B$ if and only if $V^{-T} A V^{-1} \geq D$. Let $U \in \mathbb{R}^{n \times n}$ be unitary and such that $U^{T} V^{-T} A V^{-1} U$ is diagonal (Bernstein, 2005, fact 5.8.16). Thus, $A \geq B$ if and only if $U^{T} V^{-T} A V^{-1} U \geq U^{T} D U=D$, and this last condition holds if and only if the elements of $U^{T} V^{-T} A V^{-1} U$ are not less than those of $D$. However, $A \geq 0$ implies that the elements of $U^{T} V^{-T} A V^{-1} U$ are nonnegative. Therefore,

$$
\begin{aligned}
A \geq B \quad & \Leftrightarrow \quad U^{T} V^{-T} A V^{-1} U \geq D \\
& \Leftrightarrow \quad U^{T} V^{-T} A V^{-1} U \geq \frac{1}{2}(D+|D|) \\
& \Leftrightarrow \quad A \geq \frac{1}{2} V^{T} U(D+|D|) U^{T} V \\
& =\frac{1}{2} V^{T}(D+|D|) V \\
& =[B]_{+}^{T}[B]_{+} .
\end{aligned}
$$

Lemma 17 If condition (7) holds, where $\left\{u_{t}\right\}$ and $\left\{e_{t}\right\}$ are uncorrelated (i.e. in open loop), then $[I-$ $\left.P_{u}^{\dagger} P_{u}\right] W^{T}\left(e^{j \omega}, \theta_{0}\right)=0$.
PROOF. Notice that, for every ${ }^{11} \omega \in[-\pi, \pi]$,

$$
\begin{aligned}
& {\left[I-P_{u}^{\dagger} P_{u}\right] W^{T}\left(e^{j \omega}, \theta_{0}\right)=0} \\
& \quad \Leftrightarrow \quad \mathcal{N}\left\{P_{u}^{\dagger}\right\} \perp \mathcal{R}\left\{W^{T}\left(e^{j \omega}, \theta_{0}\right)\right\} \\
& \quad \Leftrightarrow \quad \mathcal{N}\left\{P_{u}^{\dagger}\right\} \perp \mathcal{R}\left\{W^{T}\left(e^{j \omega}, \theta_{0}\right) W\left(e^{j \omega}, \theta_{0}\right)\right\} .
\end{aligned}
$$

The first equivalence follows from properties of the MoorePenrose generalised inverse (Bernstein, 2005, Theorems 2.4.3 and 6.1.6), and the second equivalence is due to the fact that the range of a matrix $A$ is equal to the range of $A A^{H}$ (Bernstein, 2005, Theorem 2.4.3).

We need to show that condition (14) holds automatically for every $\omega \in[-\pi, \pi]$. To this end, notice that, according to the partition $\theta=\left[\begin{array}{lll}\theta_{G}^{T} & \theta_{G H}^{T} & \theta_{H}^{T}\end{array}\right]^{T}$,

$$
\begin{aligned}
& P_{u}^{\dagger}=:\left[\begin{array}{c|c|c}
A_{11} & A_{12} & 0 \\
\hline A_{21} & A_{22} & 0 \\
\hline 0 & 0 & 0
\end{array}\right], \\
& \frac{\chi}{r_{\mathrm{adm}}^{2}(\omega)} T^{T}\left(e^{j \omega}, \theta_{0}\right) T\left(e^{j \omega}, \theta_{0}\right)=:\left[\begin{array}{c|c|c}
B_{11} & B_{12} & 0 \\
\hline B_{21} & B_{22} & 0 \\
\hline 0 & 0 & 0
\end{array}\right], \\
& \frac{N}{2 \pi} \int_{-\pi}^{\pi} F_{e}\left(e^{j \tau}, \theta_{0}\right) F_{e}^{H}\left(e^{j \tau}, \theta_{0}\right) d \tau=:\left[\begin{array}{c|c|c}
0 & 0 & 0 \\
\hline 0 & C_{22} & C_{23} \\
\hline 0 & C_{32} & C_{33}
\end{array}\right] .
\end{aligned}
$$

Now, by condition (7), the upper left $(2,2)$-block of $P_{u}^{\dagger}$ is non-singular, so $\mathcal{N}\left\{P_{u}^{\dagger}\right\}=\mathcal{R}\left\{[0|0| I]^{T}\right\}$. Therefore, we need to show that every vector $v=\left[0|0| \tilde{v}^{T}\right]^{T}$ is an eigenvector of $W^{T}\left(e^{j \omega}, \theta_{0}\right) W\left(e^{j \omega}, \theta_{0}\right)$ associated with the eigenvalue 0 . Let $\tilde{v}$ be an eigenvector of $C_{33}$, associated with an eigenvalue, say, $\lambda$. Since $C_{33} \geq 0$ (as $\left.\int_{-\pi}^{\pi} F_{e}\left(e^{j \tau}, \theta_{0}\right) F_{e}^{H}\left(e^{j \tau}, \theta_{0}\right) d \tau \geq 0\right)$, we have that $\lambda \geq 0$, hence

$$
\begin{aligned}
& {\left[\frac{\chi}{r_{\text {adm }}^{2}(\omega)} T^{T}\left(e^{j \omega}, \theta_{0}\right) T\left(e^{j \omega}, \theta_{0}\right)\right.} \\
& \left.-\frac{N}{2 \pi} \int_{-\pi}^{\pi} F_{e}\left(e^{j \tau}, \theta_{0}\right) F_{e}^{H}\left(e^{j \tau}, \theta_{0}\right) d \tau\right] v=\left[\begin{array}{c}
0 \\
\frac{0}{-C_{33} v}
\end{array}\right] \\
& =-\lambda v \text {, }
\end{aligned}
$$

where $-\lambda \leq 0$. Thus, by the definition of $W\left(e^{j \omega}, \theta_{0}\right)$, we have that $\bar{W}^{T}\left(e^{j \omega}, \theta_{0}\right) W\left(e^{j \omega}, \theta_{0}\right) v=0 v=0$. Since the vectors $\left[0|0| \tilde{v}^{T}\right]^{T}$, where $\tilde{v}$ is an eigenvector of $C_{33}$, span

${ }^{11} \mathcal{R}\{A\}$ and $\mathcal{N}\{A\}$ denote the range and null space of a matrix $A$, respectively (Bernstein, 2005, section 2.4). 
$\mathcal{R}\left\{[0|0| I]^{T}\right\}$, this proves (14), and this concludes the proof.

\section{References}

Bernstein, D. S., 2005. Matrix Mathematics. Princeton University Press, Princeton, New Yersey.

Bombois, X., Gevers, M., Scorletti, G., Anderson, B., 2001. Robustness analysis tools for an uncertainty set obtained by prediction error identification. Automatica 37 (10), 1629-1636.

Bombois, X., Gilson, M., 2006. Cheapest identification experiment with guaranteed accuracy in the presence of undermodeling. In: Proceedings of the 14th IFAC Symposium on System Identification (SYSID'06). Newcastle, Australia, pp. 505-510.

Bombois, X., Scorletti, G., Gevers, M., Hildebrand, R., Van den Hof, P., 2004a. Cheapest open-loop identification for control. In: Proceedings of the 43rd IEEE Conference on Decision and Control (CDC 2004). Atlantis, Bahamas, pp. 382-387.

Bombois, X., Scorletti, G., Gevers, M., Van den Hof, P., Hildebrand, R., 2006. Least costly identification experiment for control. Automatica 42 (10), 1651-1662.

Bombois, X., Scorletti, G., Van den Hof, P., 2005. Least disturbing closed-loop identification experiment for control. In: Proceedings of the 16th IFAC World Congress. Prague, Czech Republic, pp. 505-510.

Bombois, X., Scorletti, G., Van den Hof, P., Gevers, M., 2004b. Least costly identification experiment for control: A solution based on a high-order model approximation. In: Proceedings of the American Control Conference (ACC 2004). Boston, USA, pp. 2818-2823.

Boyd, S., El Ghaoui, L., Feron, E., Balakrishnan, V., 1994. Linear Matrix Inequalities in System and Control Theory. SIAM, Philadelphia, USA.

Fedorov, V. V., 1972. Theory of Optimal Experiments. Academic Press, New York and London.

Gevers, M., 2005. Identification for control: From the early achievements to the revival of experiment design. European Journal of Control 11, 1-18.

Gevers, M., Bombois, X., 2006. Input design: From open-loop to control-oriented design. In: 14th IFAC Symposium on System Identification (SYSID'06). Newcastle (Australia), pp. 13291334.

Goodwin, G. C., Payne, R. L., 1977. Dynamic System Identification: Experiment Design and Data Analysis. Academic Press, New York.

Hildebrand, R., Gevers, M., 2003. Identification for control: Optimal input design with respect to a worst-case $\nu$-gap cost function. SIAM J. Control Optim. 41 (5), 1586-1608.

Hjalmarsson, H., 2005. From experiment design to closed-loop control. Automatica 41 (3), 393-438.

Jansson, H., 2004. Experiment design with applications in identification for control. Ph.D. thesis, Royal Institute of Technology (KTH), tRITA-S3-REG-0404.

Jansson, H., Hjalmarsson, H., 2005a. Input design via lmis admitting frequency-wise model specifications in confidence regions. IEEE Transactions on Automatic Control AC-50 (10), 15341549 .

Jansson, H., Hjalmarsson, H., 2005b. Optimal experiment design in closed loop. In: 16th IFAC World Congress on Automatic Control.

Lasserre, J. B., Hiriart-Urruty, J. B., 2002. Mathematical properties of optimization problems defined by positively homogeneous functions. Journal of Optimization Theory and Applications 112 (1), 31-52.

Levadi, V. S., 1966. Design of input signals for parameter estimation. IEEE Transactions on Automatic Control AC-11 (2), 205-211.

Lighthill, M. J., 1959. Introduction to Fourier Analysis and Generalised Functions. Cambridge University Press, London.

Ljung, L., 1999. System Identification: Theory for the User, 2nd Edition. Prentice Hall, Upper Saddle River, New Jersey.

Mehra, R. K., 1974. Optimal inputs for system identification. IEEE Transactions on Automatic Control AC-19, 192-200.

Priestley, M. B., 1981. Spectral Analysis and Time Series. Academic Press, New York.

Rockafellar, R. T., 1970. Convex Analysis. Princeton University Press, Princeton, New Jersey.

Rojas, C. R., Barenthin, M., Welsh, J. S., Hjalmarsson, H., July 2008. The cost of complexity in identification of FIR systems. In: Proceedings of the 17th IFAC World Congress. Seoul, South Korea, accepted for publication.

Rojas, C. R., Welsh, J. S., Goodwin, G. C., Feuer, A., 2007. Robust optimal experiment design for system identification. Automatica 43 (6), 993-1008.

Söderström, T., Stoica, P., 1989. System Identification. Prentice Hall, Hertfordshire, United Kingdom.

Whittle, P., 1973. Some general points in the theory of optimal experimental design. J.R. Stat. Soc. 35 (1), 123-130.

Yuan, Z., Ljung, L., 1984. Black-box identification of multivariable transfer functions-asymptotic properties and optimal input design. International Journal of Control 40 (2), 233-256.

Zarrop, M., 1979. Optimal Experiment Design for Dynamic System Identification. Vol. 21 of Lecture Notes in Control and Information. Springer, Berlin, New York.

Zhou, K., Doyle, J. C., Glover, K., 1996. Robust and Optimal Control. Prentice Hall, Englewood Cliffs, New Jersey. 